\title{
Studying Extragalactic Background Fluctuations with the Cosmic Infrared Background ExpeRiment 2 (CIBER-2)
}

Alicia Lanz ${ }^{a}$, Toshiaki Arai ${ }^{b}$, John Battle $^{a}$, James Bock ${ }^{a c}$, Asantha Cooray ${ }^{d}$, Viktor Hristov ${ }^{a}$, Phillip Korngut ${ }^{c a}$, Dae Hee Lee ${ }^{e}$, Peter Mason ${ }^{a}$, Toshio Matsumoto ${ }^{b f g}$, Shuji Matsuura ${ }^{b}$, Tracy Morford $^{a}$, Yosuke Onishi ${ }^{b}$, Mai Shirahata ${ }^{h}$, Kohji Tsumura $^{i}$, Takehiko Wada ${ }^{b}$, Michael Zemcov ${ }^{a c}$

${ }^{a}$ Department of Physics, Mathematics and Astronomy, California Institute of Technology, Pasadena, CA 91125, USA;

${ }^{b}$ Department of Space Astronomy and Astrophysics, Institute of Space and Astronautical Science (ISAS), Japan Aerospace Exploration Agency (JAXA), Sagamihara, Kanagawa 252-5210, Japan;

${ }^{c}$ Jet Propulsion Laboratory (JPL), National Aeronautics and Space Administration (NASA), Pasadena, CA 91109, USA;

${ }^{d}$ Center for Cosmology, University of California, Irvine, Irvine, CA 92697, USA; ${ }^{e}$ Korea Astronomy and Space Science Institute (KASI), Daejeon 305-348, Republic of Korea; fDepartment of Physics and Astronomy, Seoul National University, Seoul 151-742, Republic of Korea;

${ }^{g}$ Institute of Astronomy and Astrophysics, Academia Sinica, Taipei 10617, Taiwan R. O. C.;

${ }^{h}$ National Institutes of Natural Science, National Astronomical Observatory of Japan (NAOJ), Tokyo 181-8588, Japan;

${ }^{i}$ Frontier Research Institute for Interdisciplinary Science, Tohoku University, Sendai, 980-8578, Japan

\begin{abstract}
Fluctuations in the extragalactic background light trace emission from the history of galaxy formation, including the emission from the earliest sources from the epoch of reionization. A number of recent near-infrared measurements show excess spatial power at large angular scales inconsistent with models of $z<5$ emission from galaxies. These measurements have been interpreted as arising from either redshifted stellar and quasar emission from the epoch of reionization, or the combined intra-halo light from stars thrown out of galaxies during merging activity at lower redshifts. Though astrophysically distinct, both interpretations arise from faint, low surface brightness source populations that are difficult to detect except by statistical approaches using careful observations with suitable instruments. The key to determining the source of these background anisotropies will be wide-field imaging measurements spanning multiple bands from the optical to the near-infrared.

The Cosmic Infrared Background ExpeRiment 2 (CIBER-2) will measure spatial anisotropies in the extragalactic infrared background caused by cosmological structure using six broad spectral bands. The experiment uses three 2048 x 2048 Hawaii-2RG near-infrared arrays in three cameras coupled to a single $28.5 \mathrm{~cm}$ telescope housed in a reusable sounding rocket-borne payload. A small portion of each array will also be combined with a linear-variable filter to make absolute measurements of the spectrum of the extragalactic background with high spatial resolution for deep subtraction of Galactic starlight. The large field of view and multiple spectral bands make CIBER-2 unique in its sensitivity to fluctuations predicted by models of lower limits on the luminosity of the first stars and galaxies and in its ability to distinguish between primordial and foreground anisotropies. In this paper the scientific motivation for CIBER-2 and details of its first flight instrumentation will be discussed, including detailed designs of the mechanical, cryogenic, and electrical systems. Plans for the future will also be presented.
\end{abstract}

Send correspondence to A.L.: E-mail: alicialanz@caltech.edu, Telephone: 16263952016

Space Telescopes and Instrumentation 2014: Optical, Infrared, and Millimeter Wave, edited by Jacobus M. Oschmann, Jr., Mark Clampin, Giovanni G. Fazio, Howard A. MacEwen, Proc. of SPIE Vol. 9143, 91433N · C 2014 SPIE CCC code: $0277-786 \mathrm{X} / 14 / \$ 18 \cdot$ doi: $10.1117 / 12.2057304$ 


\section{INTRODUCTION}

The near-infrared extragalactic background light (EBL) is composed of all photons emitted from stars throughout cosmic history $^{1}$. The rest-frame optical and ultra-violet radiation from early objects, emitted during reionization of the universe $(z>7)$, has a unique spectral feature that is potentially observable in the near-infrared EBL today $^{2}$. Prior to reionization, the universe was filled with neutral hydrogen that absorbed photons at wavelengths above the Lyman-alpha cutoff of $0.1216 \mu \mathrm{m}$. This suggests that the radiation from early galaxies responsible for reionization can be identified by a characteristic spectral feature where a signal is present at wavelengths longer than the Lyman limit and absent at shorter wavelengths. Assuming reionization occurred prior to $z \sim 7^{3}$, the this feature is observable today at wavelengths longer than $0.9 \mu \mathrm{m}$.

Direct observation of the mean intensity of the near-infrared EBL with absolute photometry has proven challenging due to bright foregrounds, especially Zodiacal Light reflected off dust particles in our solar system ${ }^{1}$, so searching for this feature in an absolute measurement would be extremely challenging. Spatial fluctuation measurements are able to discriminate EBL signals from foregrounds by taking advantage of the differing spatial structure of the reionization signal and the foregrounds ${ }^{1,4-6}$.

Recent spatial fluctuation observations report levels far in excess of the level predicted by local $(z<5)$ galaxy counts ${ }^{1,7-9}$. Early galaxies must contribute to this excess to some extent. In addition to early galaxies, EBL fluctuations can also arise from intrahalo light (IHL) ${ }^{9}$ generated by diffusely distributed stars expelled from galaxies during structure formation at $0<z<2$. Emission from IHL is expected to have a spectral shape that peaks near $1.5 \mu \mathrm{m}$ and slowly decreases, in contrast to the sharp cutoff expected in the spectral features of early galaxies. Spectral decomposition between multiple wavelength bands is key to differentiating between these contributions to large scale EBL fluctuations.

An instrument designed to observe spatial fluctuations in the near-infrared EBL and separate out contributing components should include the design features included in Table 1. The Cosmic Infrared Background ExpeR-

Table 1. CIBER-2 Design Drivers

\begin{tabular}{|l|l|}
\hline Science Driver & Design Feature \\
\hline \hline Separation of local astrophysical foregrounds. & $\begin{array}{l}\text { Spatial fluctuations measurements at degree angular scales, } \\
\text { observed above the atmosphere. }\end{array}$ \\
\hline Masking foreground galaxies. & $\begin{array}{l}4^{\prime \prime} \times 4^{\prime \prime} \text { pixel size, so that the image of galaxies is small } \\
\text { compared to a pixel. }\end{array}$ \\
\hline Component separation. & $\begin{array}{l}\text { Simultaneous multi-band observations spanning the optical } \\
\text { and near-infrared. }\end{array}$ \\
\hline Comparison to previous datasets. & $\begin{array}{l}\text { Observational fields selected to overlap with previous mea- } \\
\text { surements. }\end{array}$ \\
\hline
\end{tabular}

iment 2 (CIBER-2) is a wide field, degree-scale camera in a sounding rocket payload specifically designed for EBL spatial fluctuations measurements in six near-infrared and optical wavelength bands. In a short sounding rocket flight, CIBER-2 has sufficient sensitivity to probe to the faint signal level predicted for galaxies from the epoch of reionization, as shown in Figure 1. This paper presents the design of the CIBER-2 hardware and plans for CIBER-2 flights.

\section{THE CIBER-2 PAYLOAD}

CIBER-2 consists of a Cassegrain telescope assembly, imaging optics, and cryogenics mounted aboard a sounding rocket. Two dichroic beam-splitters spectrally subdivide the incident radiation into three optical paths, which are further subdivided in two wavelength bands per path, for a total of six observational wavelength bands. These systems are shown in Figure 2 and described in detail below. 


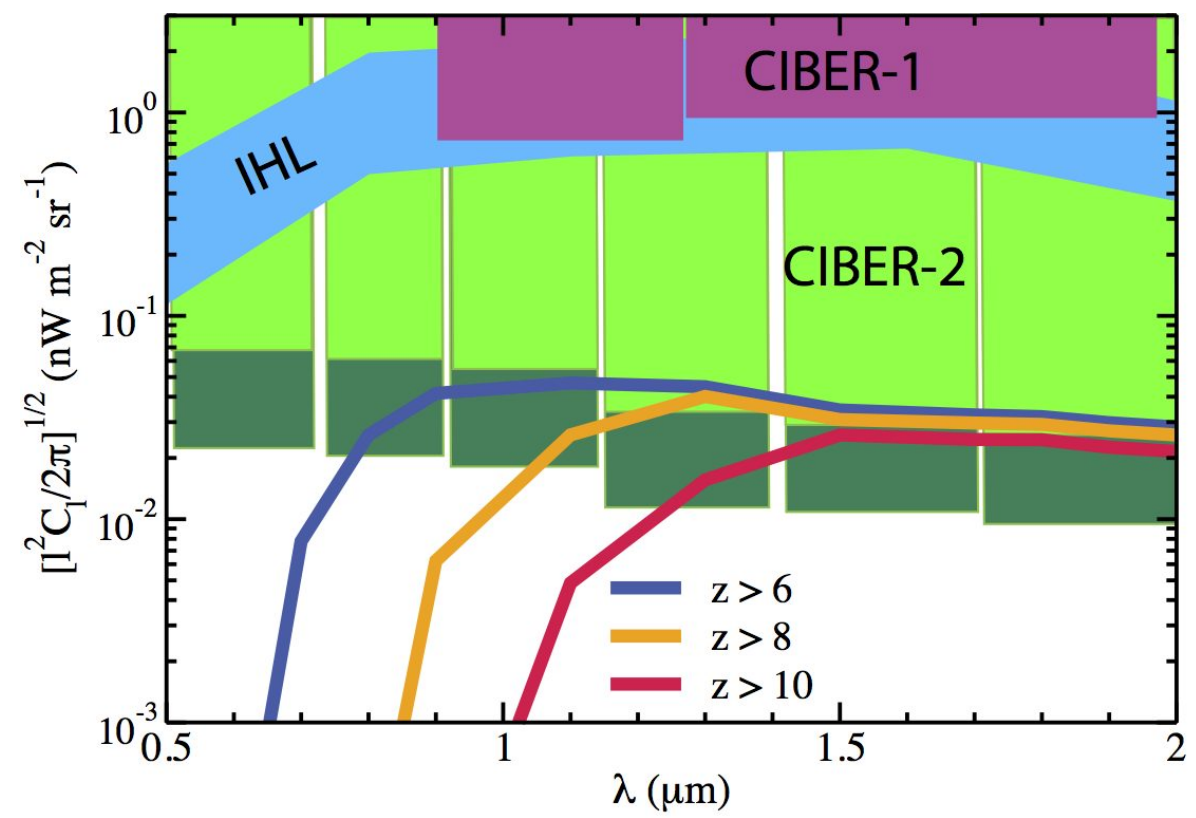

Figure 1. Depth of CIBER-2 fluctuation measurements in six wavelength bands from 0.5-2.0 $\mu$ m at a particular angular scale. Light green indicates the depth achieved by CIBER-2 in a single flight in one field. Dark green indicates depth from all data in four flights. The light blue band indicates the predicted level of IHL across the wavelength range ${ }^{10}$. The three thin lines show the expected contribution to EBL fluctuations from early galaxies assuming different reionization histories, with the dark blue line (left) for $z>6$, yellow line (middle) for $z>7$, and red line (right) for $z>8$. Also shown in purple is the depth from the second and third flights of CIBER-1 in each of its two wavelength bands for comparison.

\subsection{Sounding Rocket Constraints}

The CIBER-2 sounding rocket has a fixed outer diameter of 17.26" within which the instrument payload must fit $^{11}$. The telescope assembly and imaging optics are housed in an evacuated segment of the rocket, and are cooled to $\sim 80 \mathrm{~K}$ by an on-board liquid nitrogen reservoir. This payload section is capped at the aft end of the rocket, toward the motors, by a door that opens to the telescope aperture.

\subsection{Telescope Assembly}

The CIBER-2 Cassegrain telescope has a $28.5 \mathrm{~cm}$ primary mirror and $14 \mathrm{~cm}$ secondary mirror. The primary mirror is attached to a support plate with flexures. The secondary mirror is held up by a support spider that mounts to the support plate. A cylindrical optical baffle that provides both structural support and light blocking is also attached to the support plate. Titanium flexures fix the optical baffle to a mounting ring that is attached directly to the rocket skin. These flexures relieve mechanical stress and provide a thermal break between the rocket skin and the telescope assembly.

\subsection{Imaging Optics}

The imaging optics are mounted to an optical bench that connects the primary mirror support plate and the cryogenic tank. Light from the secondary mirror passes through the Cassegrain hole to the imaging optics section, where it is focused by a field lens. Next, two beam splitters direct the incoming light into three paths, each with a 2048 x 2048 HAWAII-2RG detector array. Additional filtering splits the light into two wavelength bands per detector array, resulting in a total of six distinct wavelength bands spanning the range of $0.5<\lambda<2.0 \mu \mathrm{m}$. Each of the three light paths is very similar, consisting of a beam splitter, bend mirror(s), a collimator lens, a band pass filter, a camera lens, and a focal plane assembly that contains a detector array. Figure 3 shows a representative ray tracing diagram including all of the optical components. The imaging optics and mirrors are manufactured to the specifications of the science team by Genesia Corporation of Japan. All of the optical 


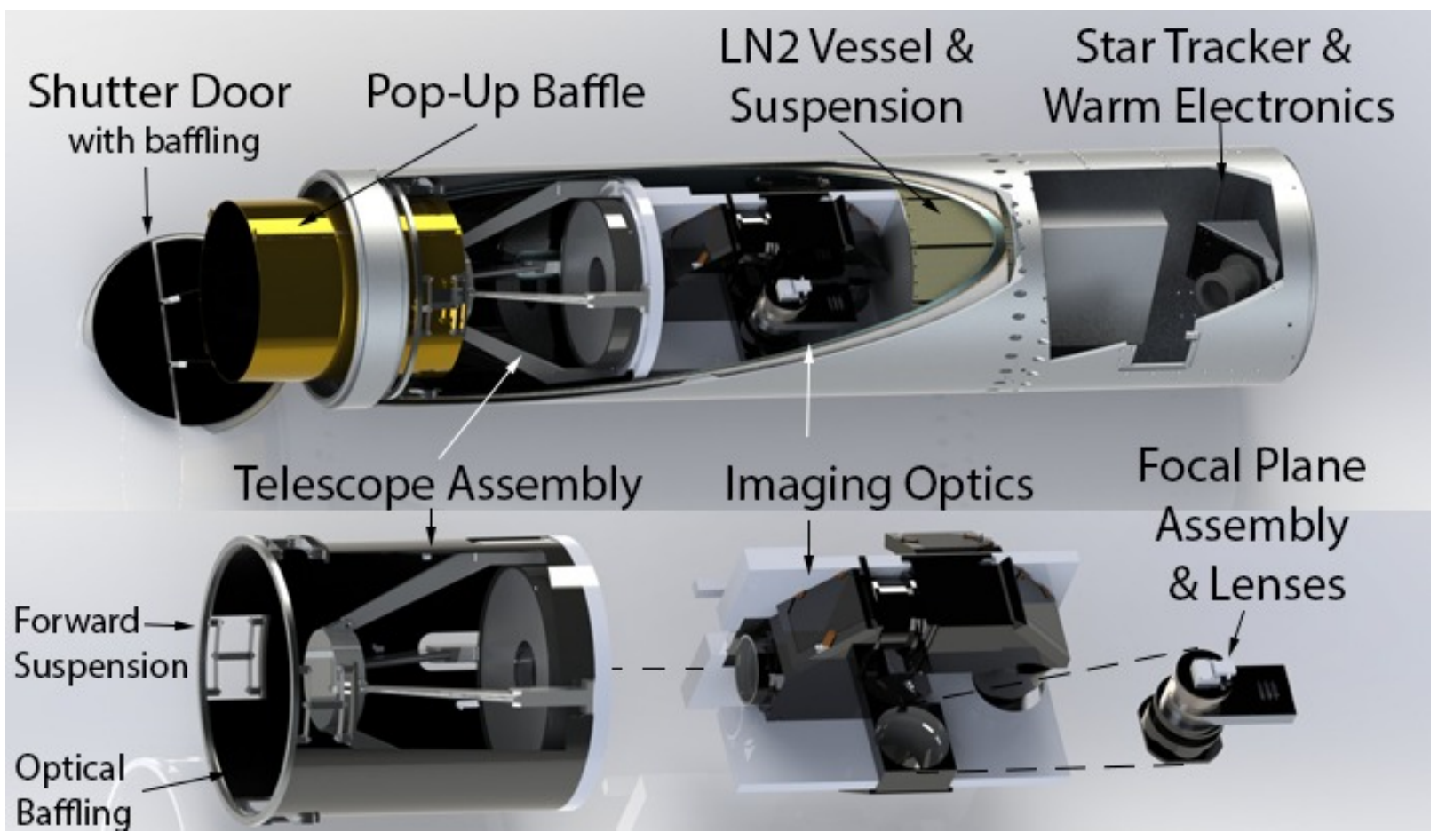

Figure 2. Solid model of the CIBER-2 instrument. A $28.5 \mathrm{~cm}$ Cassegrain telescope directs light into the imaging optics, where beam splitters divide the light into three optical paths. Each optical path travels to one of three focal plane assemblies, where a broadband filter subdivides the light into two wavelength bands which are both recorded by a single detector array for a total of six band. A small segment of each detector array is also covered linear-variable filter. The imaging optics are mounted to an optical bench that connects the Cassegrain telescope assembly to a liquid nitrogen cryostat. Radiative shielding is provided by a radiatively-cooled door liner and cryogenically-cooled pop-up baffle that extends during observations. A section forward of the instrument section contains an electronics box and a star tracker.

components are supported by flexures or spring retainers to handle the thermal compression stress in cooling and to maintain optical alignment against vibration and shock during launch.

Table 2 lists the optical parameters of the telescope, along with sensitivities and spectral band information. Sensitivities in Table 2 assume a diffuse sky brightness 1.5 times the brightness observed by DIRBE at the north ecliptic pole ${ }^{12}$, include all detector and photon noise contributions assuming line fitting to constant radiance array reads, and allocate 35 seconds per integration, times two roll angles, for a total integration time of 70 seconds per field. We compute sensitivities using a model for the instrument ${ }^{13}$,

$$
\delta \lambda I_{\lambda}=\delta i_{\text {total }}\left(\frac{h \nu}{(\Delta \lambda / \lambda) \eta \mathrm{A} \Omega}\right)\left[\mathrm{nW} \mathrm{m} \mathrm{m}^{-2} \mathrm{sr}^{-1}\right]
$$

where $\nu I_{\nu}$ is the sky brightness, $\delta i_{\text {total }}$ is the quadrature sum of the photon noise and read noise, $h \nu$ is the average energy per photon, $\frac{\Delta \lambda}{\lambda}$ is the fractional bandwidth, $A \Omega$ is the observing area, and $\eta$ is the total optical and quantum efficiency of the instrument.

Focal plane assemblies provide housing for the detector arrays, band defining filters, and a circuit board for the first stage of detector readout, as shown in Figure 4. The detector arrays are thermally stood off from the optical assembly by a Molybdenum support structure, limiting the rate of temperature change below $2 \mathrm{~K} / \mathrm{min}$ during cooling and allowing for the inclusion of a thermal regulation feedback system to stabilize the focal plane temperature during flight. The thermal stabilization system consists of a heating resistor and thermometer mounted to the focal plane, with the amount of power dissipated by the heater controlled by a proportionalintegral-derivative (PID) loop. A filter assembly is mounted to the Molybdenum support structure, suspending 


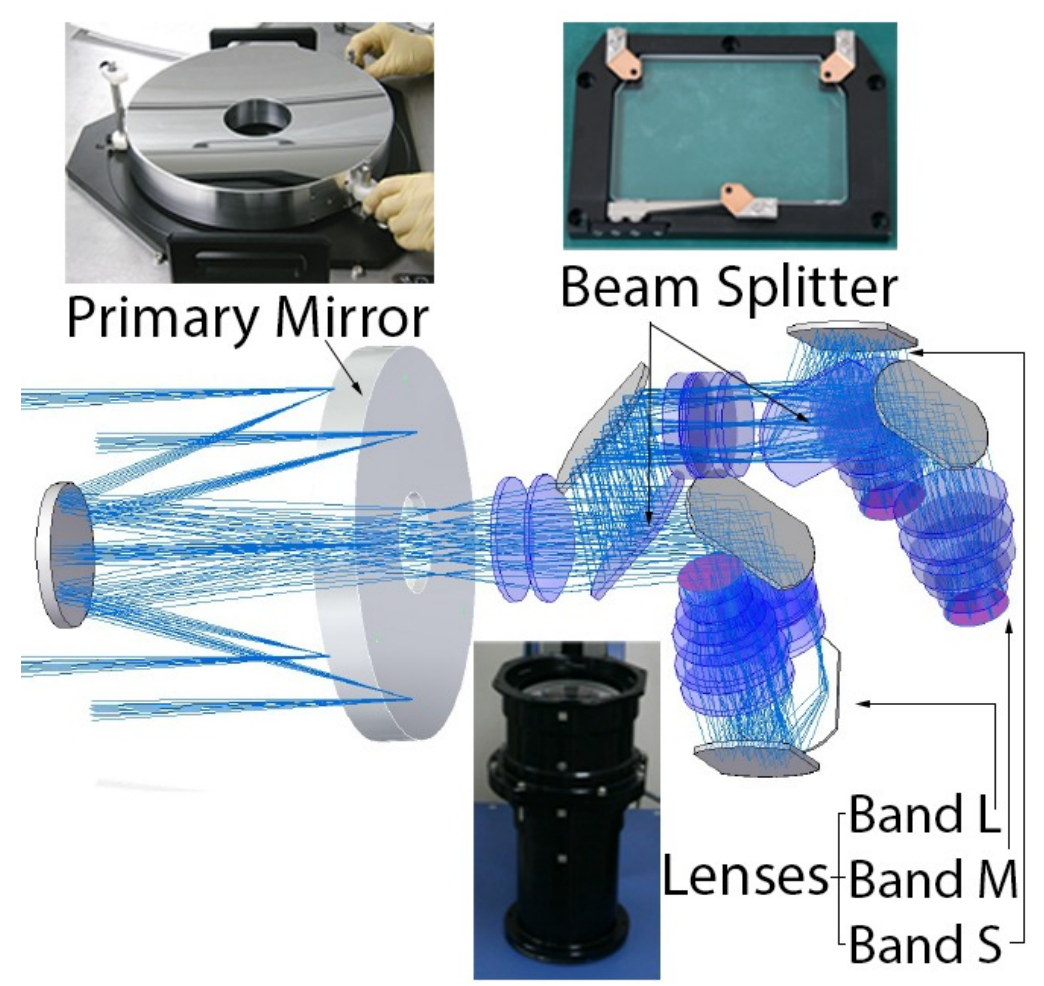

Figure 3. Schematic representation of the CIBER-2 light paths. Incoming light is split into three light paths using two dichroic beam splitters. Bend mirrors, a collimator lens, a band pass filter, and a camera lens direct the light to a focal plane assembly that contains a detector array. Photographs of fabricated components are also shown.

Table 2. CIBER-2 Instrument Parameters

\begin{tabular}{|c|c|c|c|c|c|c|c|}
\hline Parameter & \multicolumn{6}{|c|}{ CIBER-2 } & Units \\
\hline Aperture & \multicolumn{6}{|c|}{28.5} & $\mathrm{~cm}$ \\
\hline Pixel Size & \multicolumn{6}{|c|}{4} & $\operatorname{arcsec}$ \\
\hline Array & \multicolumn{6}{|c|}{$\mathrm{HgCdTe}$} & \\
\hline Format & \multicolumn{6}{|c|}{$2048 \times 2048$} & \\
\hline Field of View & \multicolumn{6}{|c|}{$1.1 \times 2.2$ for imager bands, 0.4 for LVF } & degrees \\
\hline Dark Current & \multicolumn{6}{|c|}{$<0.1$} & $\mathrm{e}-/ \mathrm{s}$ \\
\hline Read Noise (CDS) & \multicolumn{6}{|c|}{12} & e- \\
\hline Band & 1 & 2 & 3 & 4 & 5 & 6 & \\
\hline$\lambda$ & 0.600 & 0.800 & 1.030 & 1.280 & 1.550 & 1.850 & $\mu \mathrm{m}$ \\
\hline$\frac{\delta \lambda}{\lambda}$ & 0.33 & 0.25 & 0.24 & 0.20 & 0.20 & 0.16 & \\
\hline Array QE & 0.90 & 0.80 & 0.83 & 0.81 & 0.82 & 0.82 & \\
\hline Optics QE & 0.75 & 0.73 & 0.81 & 0.85 & 0.87 & 0.87 & \\
\hline Photocurrent & 9.5 & 6.8 & 8.1 & 7.8 & 7.7 & 3.8 & $\mathrm{e}-/ \mathrm{s}$ \\
\hline$\nu I_{\nu}(\mathrm{sky})$ & 525 & 450 & 400 & 380 & 320 & 224 & $\mathrm{nW} \mathrm{m}^{-2} \mathrm{sr}^{-1}$ \\
\hline$\delta \nu I_{\nu}(1 \sigma /$ pixel $)$ & 38.0 & 44.8 & 33.9 & 30.6 & 25.0 & 23.0 & $\mathrm{nW} \mathrm{m}^{-2} \mathrm{sr}^{-1}$ \\
\hline$\delta F_{\nu}(3 \sigma)$ & 21.5 & 21.1 & 21.0 & 21.0 & 21.0 & 20.9 & $\mathrm{AB}$ mag \\
\hline
\end{tabular}

a set of band defining filters and a linear-variable filter (LVF) above the detector array. Band defining filters divide the incoming beam into two wavelength bands for imaging. A linear-variable filter covers a small area of each detector array for absolute spectrophotometeric measurements, co-located with a subset of dark pixels for dark current reference measurements. The detector array and filter assembly are encased in an aluminum assembly. This assembly also houses a focal plane board for read out of the detector electronics. 


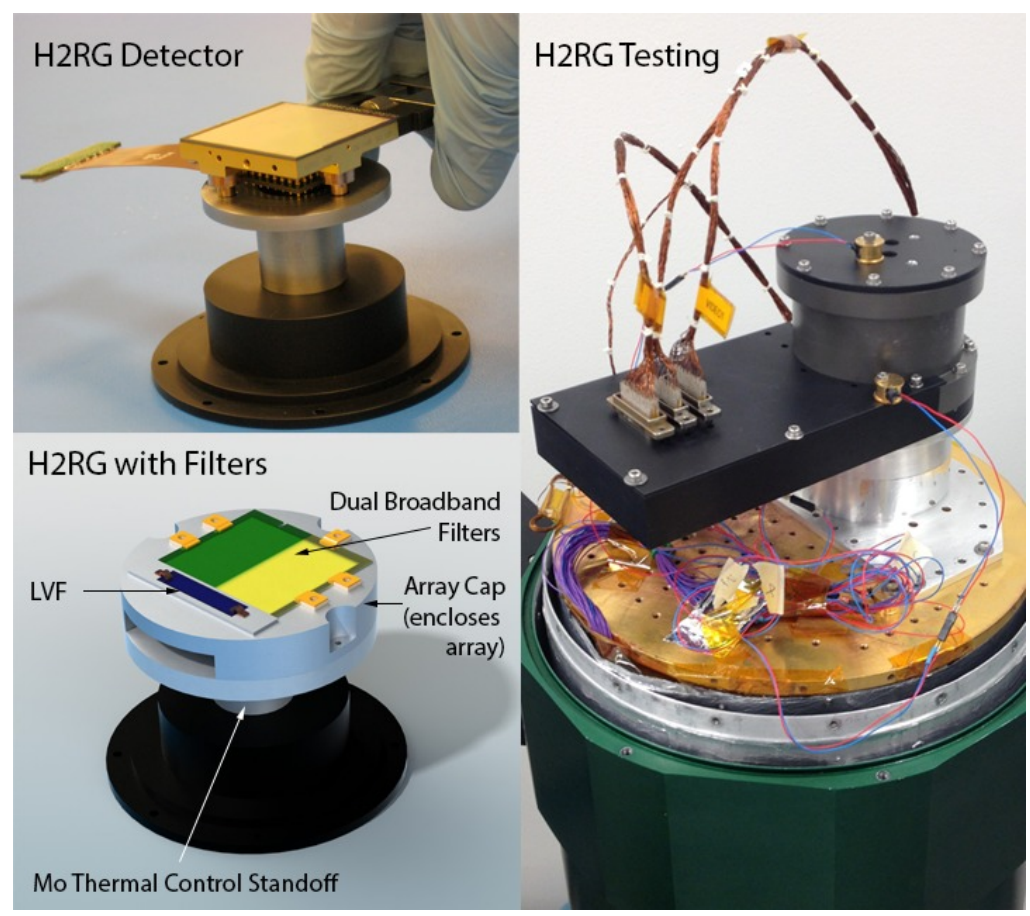

Figure 4. Counterclockwise from top left: H2RG detector array undergoing installation onto a Molybdenum support structure; solid model of filter assembly, with array cap supporting a dual broadband filter and a linear-variable filter (LVF); and full focal plane assembly mounted in a cryostat for testing of noise properties.

For calibration purposes, the payload also contains a cold shutter and calibration lamps. The cold shutter is mounted to the forward face of the primary mirror support plate. It allows for measurement of the dark current before and during flight. The calibration lamps are housed within an assembly attached to the optical bench. An optical fiber carries the light from the calibration lamp assembly to a mounting point near the center of the secondary mirror. These are used to illuminate the light path with a known, fixed brightness for reference observational frames used to track the relative gain of individual detectors.

\subsection{Cryogenics}

The CIBER-2 cryogenic system is a duplicate of the successful cryogenic system used in CIBER-1 ${ }^{14}$. This system consists of a 7 liter liquid nitrogen vessel filled with an open-cell aluminum foam that ensures thermal contact between liquid and and metal in zero-gravity conditions. The optical bench supporting the imaging optics are mounted directly to the cryostat. G-10 plates mount the cryostat to the thermal vacuum bulkhead that marks the forward boundary of the vacuum payload. This bulkhead has hermetic connections for cabling to travel from the vacuum payload section to the warm electronics section, where the electronics described in Section 3 are located.

\subsection{Radiative Shielding}

The telescope assembly, imaging optics, and cryogenic system are enclosed in a radiation shield made of Aluminum 1100, mounted to the cryostat for cooling. The radiation shield buffers the cryogenically cooled instruments from radiation from the skin. The radiation shield is wrapped in a Mylar blanket to further reduce the radiative load. A pop-up baffle provides additional shielding from stray light from the rocket skin and shutter door. This pop-up baffle is stowed inside the telescope assembly upon ascent and descent, and deployed to extend beyond the lip of the skin during observations. The shutter door has additional blackened baffling covers that are stood off from the door itself with Vespel posts. 


\section{DETECTORS AND ELECTRONICS}

The CIBER-2 electronics chain carries signals between the detectors, the experiment housekeeping, and the rocket telemetry system. It is made up of the circuit boards located near the detectors in the focal plane assemblies described in Section 2.3 and three other board types located in the star tracker section of the rocket, forward of the experiment section: data acquisition boards, array processing and housekeeping boards, and data storage boards.

CIBER-2 has three 2048 x 2048 HAWAII-2RG (H2RG) detector arrays, which are second-generation commercial HgCdTe near-IR detectors manufactured by Teledyne Scientific and Imaging Corporation. Each detector is biased and read out by custom electronics on the focal plane boards. The 2048 columns of the detector array are divided into 32 channels, which are read out in "slow" mode to reduce readout time and noise. As the pixel voltages have a large offset, an external reference voltage close to the average pixel voltage is used to remove the offset. A reference pixel row will be read out periodically during the full detector array readout, providing a method of $1 / \mathrm{f}$ noise mitigation ${ }^{15}$.

Two data acquisition boards per detector array digitize the read out voltages. Each data acquisition board handles 20 channels; 16 channels for detector output and 4 channels for associated housekeeping output. The signals from both data acquisition boards are passed to a single array processing and housekeeping board, which temporarily stores the data locally before passing it to the data storage board for permanent storage. The array processing and housekeeping boards also perform clocking and command functions for the detector array, pass housekeeping and status data to a NASA telemetry system, and respond to signals from external NASA systems.

The data rate required for three detectors exceeds the capabilities of the NASA ground telemetry systems. Instead of transmitting all data and housekeeping down to the ground station, CIBER-2 stores flight data onboard and transmits only a small fraction for diagnostic purposes. Solid-state storage is required to withstand the physical environment experienced in flight. Each detector array requires one data storage board with two 128 GB flash memory chips. This data storage board receives the digitized detector array output data from the array processing and housekeeping board and stores a primary and backup copy on each of the flash memory chips. Housekeeping data and a single channel of flight data are transmitted via rocket telemetry systems to the ground station. Raw rocket telemetry received by the ground system is forwarded to custom ground station elections (GSE) which displays the data in real time. The GSE system is also used during testing.

\section{ROCKET PAYLOAD}

The NASA Sounding Rocket Program is described in Reference 11. The Sounding Rocket program provides standardized systems for launch, payload separation, active pointing during flight, real time telemetry, and parachute recovery. CIBER-2 is designed to launch on the Black Brant IX vehicle system, consisting of a Mark 70 Terrier booster stage and a Black Brant sustainer. These motors make up the aft end of the rocket. Moving forward, the entire rocket consists of a payload separation system, the CIBER-2 experiment (Sections 2.2-2.5), a star tracker section that contains the CIBER-2 warm electronics (Section 3), a telemetry section, an active guidance system, an attitude control system, and finally the ogive recovery system.

The CIBER-2 launch sequence closely follows the launch sequence of CIBER-1 flights 1 through $3^{14}$. The Mark 70 Terrier rocket motor burns for approximately 6 seconds for launch. This segment has guide fins that cause the rocket to spin at a rate of approximately $4 \mathrm{~Hz}$, providing thrust axis stability. Once burn is complete, the motor is separated from the rest of the rocket by drag. The second stage burns for approximately 30 seconds then is ejected from the remaining rocket sections by the payload separation section. Next, cables with weighted ends are released from the payload section to reduce the spin rate of the rocket to a rate of approximately 0.5 $\mathrm{Hz}$. Once the lower spin rate is achieved, the cables are released. The telescope aperture opens and observations begin. Upon completion of observations and prior to re-entry, the telescope aperture is closed. Gas expelled from the attitude control system is used to increase the rocket spin rate to approximately $1 \mathrm{~Hz}$. The rocket re-enters the atmosphere and the parachute deploys when the rocket reaches a height of 16,000 feet. The payload has a velocity of approximately $30 \mathrm{ft} / \mathrm{s}$ when it collides with the ground. Recovery of the payload begins several hours after completion of flight. Payload recovery is crucial for CIBER-2, as the data collected in flight will be stored on the rocket. 


\section{FUTURE PLANS AND SCIENCE EXTRACTION}

CIBER-2 plans two flights in the configuration described in this paper. The first flight is planned for 2015 with the second flight to follow six months later. The flights are separated by six months for consistency checks on absolute photometry measurements with the LVF spectrometer, as the Zodiacal foreground is modulated by the Earth's annual path around the sun. Two flights will also allow for cross correlation between CIBER-2 flight data to characterize and eliminate instrumental artifacts. Additional flights are planned with different optical configurations, such as modified wavebands.

CIBER-2 observes five fields during a flight ${ }^{16}$. These fields are chosen to coincide with previous optical and spatial fluctuation observations ${ }^{7-9}$ for cross correlation and point source removal. Additionally, launch windows are defined so that the sun and moon are below the depressed horizon ${ }^{16}$. Typical candidate fields are Bootes, North Ecliptic Pole, Lockman Hole, and ELAIS-N1. CIBER-2 observes each field for 35 seconds at two different roll angles to center each of the wavelength bands on the same sky. Each field thus has a total observation time of 70 seconds per field, used to calculate the sensitivities in Table 2.

CIBER-2 data is processed by a custom data analysis pipeline ${ }^{10,17}$. Raw data retrieved from the rocket payload is a time stream that must be converted into detector array images, calibrated, flat-field corrected, and aligned on the sky. Bright astronomical sources (J-band $\sim 19 \mathrm{AB}$ magnitude) are masked, as are any residual source structure, such as diffraction spikes due to CIBER-2's secondary mirror support structures. The power spectrum of a sky image is then generated. Contributions to the power spectrum from instrumental and photon noise, the beam transfer function, and the effects of mode coupling due to masking of sources are then modeled and quantified. As CIBER-2 observes in six spectral bands, a total of 21 auto- and cross-spectra are available for science analysis. Various contributions to the large scale EBL fluctuations will be identified from spectral decomposition of the auto- and cross-spectra.

\section{ACKNOWLEDGMENTS}

This work was supported by NASA APRA research grants NNX07AI54G, NNG05WC18G, NNX07AG43G, NNX07AJ24G, and NNX10AE12G. Japanese participation in CIBER was supported by KAKENHI (2034, 18204018, 19540250, 21340047 and 21111004) from Japan Society for the Promotion of Science (JSPS) and the Ministry of Education, Culture, Sports, Science and Technology (MEXT). Korean participation in CIBER was supported by the Pioneer Project from Korea Astronomy and Space Science Institute (KASI). M.Z. and P.K. acknowledge support from NASA Postdoctoral Program Fellowships, A.C. acknowledges support from an NSF CAREER award AST-0645427 and NSF AST-1313319, and K.T. acknowledges support from the JSPS Research Fellowship for Young Scientists.

\section{REFERENCES}

[1] Hauser, M. G. and Dwek, E., "The cosmic infrared background: Measurements and implications," Annual Reviews of Astronomy and Astrophysics 39, 249-307 (2001).

[2] Santos, M. R., Bromm, V., and Kamionkowski, M., "The contribution of the first stars to the cosmic infrared background," Monthly Notice of the Royal Astronomical Society 336, 1082-1092 (November 2002).

[3] Hinshaw, G., Larson, D., Komatsu, E., Spergel, D. N., Bennett, C. L., Dunkley, J., Nolta, M. R., Halpern, M., Hill, R. S., Odegard, N., Page, L., Smith, K. M., Weiland, J. L., Gold, B., Jarosik, N., Kogut, A., Limon, M., Meyer, S. S., Tucker, G. S., Wollack, E., and Wright, E. L., "Nine-year Wilkinson Microwave Anisotropy Probe (WMAP) observations: Cosmological parameter results," The Astrophysical Journal Supplement 208 (2013).

[4] Kashlinsky, A., Arendt, R., Gardner, J. P., Mather, J. C., and Moseley, S. H., "Detecting population III stars through observations of near-infrared cosmic infrared background anisotropies," The Astrophysical Journal 608, 1-9 (June 2004).

[5] Cooray, A., Bock, J. J., Keatin, B., Lange, A. E., and Matsumoto, T., "First star signature in infrared background anisotropies," The Astrophysical Journal 606(2) (2004).

[6] Cooray, A., Gong, Y., Smidt, J., and Santos, M. G., "The near-infrared background intensity and anisotropies during the epoch of reionization," The Astrophysical Journal 756, 15 (September 2012). 
[7] Kashlinsky, A., Arendt, R. G., Mather, J., and Moseley, S. H., "New measurements of cosmic infrared background fluctuations from early epochs," The Astrophysical Journal 654, L5-L8 (January 2007).

[8] Kashlinsky, A., Arendt, R. G., Ashby, M. L. N., Fazio, G. G., Mather, J., and Moseley, S. H., "New measurements of the cosmic infrared background fluctuations in deep Spitzer/IRAC survey data and their cosmological implications," The Astrophysical Journal 753 (July 2012).

[9] Cooray, A., Smidt, J., De Bernardis, F., Gong, Y., Stern, D., Ashby, M. L. N., Eisenhardt, P. R., Frazer, C. C., Gonzalez, A. H., Kochanek, C. S., Kozłowski, S., and Wright, E. L., "Near-infrared background anisotropies from diffuse intrahalo light of galaxies," Nature 494, 514-517 (October 2012).

[10] Zemcov, M., Smidt, J., Arai, T., Bock, J., Cooray, A., Gong, Y., Kim, M. G., Korngut, P. M., Lam, A. C., Lee, D. H., Matsumoto, T., Matsuura, S., Nam, U. W., Roudier, G., Tsumura, K., and Wada, T., "Origin of near-infrared extragalactic background light anisotropy," submitted (2014).

[11] NASA Sounding Rockets Program Office, The NASA Sounding Rocket Program Handbook.

[12] Kelsall, T., Weiland, J. L., Franz, B. A., Reach, W. T., Arendt, R. G., Dwek, E., Freudenreich, H. T., Hauser, M. G., Moseley, S. H., Odegard, N. P., Silverberg, R. F., and Wright, E. L., "The COBE Diffuse Infrared Background Experiment search for the cosmic infrared background. II. Model of the interplanetary dust cloud," The Astrophysical Journal 508, 44-73 (November 1998).

[13] Garnett, J. D. and Forrest, W. J., "Multiply sampled read-limited and background-limited noise performance," Proc. SPIE 1946 (October 1993).

[14] Zemcov, M., Arai, T., Battle, J., Bock, J., Cooray, A., Hristov, V., Keating, B., Kim, M. G., Lee, D. H., Levenson, L. R., Mason, P., Matsumoto, T., Matsuura, S., Nam, U. W., Renbarger, T., Sullivan, I., Suzuki, K., Tsumura, K., and Wada, T., "The Cosmic Infrared Background ExpeRiment (CIBER): A sounding rocket payload to study the near infrared extragalactic background light," The Astrophysical Journal Supplement Series 207 (Aug 2013).

[15] Moseley, S. H., Arendt, R. G., Fixsen, D. J., Lindler, D., Loose, M., and Rauscher, B. J., "Reducing the read noise of H2RG detector arrays: eliminating correlated noise with efficient use of reference signals," Proc. SPIE $\mathbf{7 7 4 2}$ (2010).

[16] Tsumura, K., Battle, J., Bock, J., Cooray, A., Hristov, V., Keating, B., Lee, D. H., Levenson, L. R., Mason, P., Matsumoto, T., Matsuura, S., Nam, U. W., Renbarger, T., Sullivan, I., Suzuki, K., Wada, T., and Zemcov, M., "Observations of the near-infrared spectrum of the Zodiacal Light with CIBER," The Astrophysical Journal 719, 394-402 (August 2010).

[17] Bock, J., Sullivan, I., Arai, T., Battle, J., Cooray, A., Hristov, V., Keating, B., Kim, M. G., Lam, A. C., Lee, D. H., Levenson, L. R., Mason, P., Matsumoto, T., Matsuura, S., Mitchell-Wynne, K., Nam, U. W., Renbarger, T., Smidt, J., Suzuki, K., Tsumura, K., Wada, T., and Zemcov, M., "The Cosmic Infrared Background ExpeRiment (CIBER): The wide-field imagers," The Astrophysical Journal Supplement Series 207 (Aug 2013).

[18] Madau, P. and Silk, J., "Population III and the near-infrared background excess," Monthly Notices of the Royal Astronomical Society: Letters 359(1) (2005).

[19] Matsuura, S., Shirahata, M., Kawada, M., Takeuchi, T. T., Burgarella, D., Clements, D. L., Jeong, W.-S., Hanami, H., Khan, S. A., Matsuhara, H., Nakagawa, T., Oyabu, S., Pearson, C. P., Pollo, A., Serjeant, S., Takagi, T., and White, G. J., "Detection of the cosmic far-infrared background in AKARI deep field south," The Astrophysical Journal 737 (August 2011).

[20] Matsumoto, T., Seo, H. J., Jeong, W.-S., Lee, H. M., Matsuura, S., Matsuhara, H., Oyabu, S., Pyo, J., and Wada, T., "AKARI observation of the fluctuation of the near-infrared background," The Astrophysical Journal 742 (December 2011).

[21] Matsumoto, T., Matsuura, S., Murakami, H., Tanaka, M., Freund, M., Lim, M., Cohen, M., Kawada, M., and Noda, M., "Infrared telescope in space observations of the near-infrared extragalactic background light," The Astrophysical Journal 626, 31-43 (June 2005).

[22] Dwek, E. and Arendt, R. G., "A tentative detection of the cosmic infrared background at 3.5 microns from COBE/DIRBE observations," The Astrophysical Journal 508, L9-L12 (November 1998). 
[23] Hauser, M. G., Arendt, R. G., Kelsall, T., Dwek, E., Odegard, N., Weiland, J. L., Freudenreich, H. T., Reach, W. T., Silverberg, R. F., Moseley, S. H., Pei, Y. C., Lubin, P., Mather, J. C., Shafer, R. A., Smoot, G. F., Weiss, R., Wilkinson, D. T., and Wright, E. L., "The COBE Diffuse Infrared Background Experiment search for the cosmic infrared background. I. Limits and detections," The Astrophysical Journal 508, 25-43 (November 1998).

[24] Korngut, P. M., Renbarger, T., Arai, T., Battle, J., Bock, J., Brown, S. W., Cooray, A., Hristov, V., Keating, B., Kim, M. G., Lanz, A., Lee, D. H., Levenson, L. R., Lykke, K., Mason, P., Matsumoto, T., Matsuura, S., Nam, U. W., Shultz, B., Smith, A. W., Sullivan, I., Tsumura, K., Wada, T., and Zemcov, M., "The Cosmic Infrared Background ExpeRiment (CIBER): the narrow band spectrometer," The Astrophysical Journal Supplement Series 207, 34 (Aug 2013).

[25] Tsumura, K., Arai, T., Battle, J., Bock, J., Brown, S., Cooray, A., Hristov, V., Keating, B., Kim, M. G., Lee, D. H., Levenson, L. R., Lykke, K., Mason, P., Matsumoto, T., Matsuura, S., Murata, K., Nam, U. W., Renbarger, T., Smith, A., Sullivan, I., Suzuki, K., Wada, T., and Zemcov, M., "The Cosmic Infrared Background ExpeRiment (CIBER): The low resolution spectrometer," The Astrophysical Journal Supplement Series 207 (2012).

[26] Helgason, K., Ricotti, M., and Kashlinsky, A., "Reconstructing the near-IR background fluctuations from known galaxy populations using multiband measurements of luminosity functions," The Astrophysical Journal 752, 18 (June 2012).

[27] Kashlinsky, A., Arendt, R. G., Mather, J., and Moseley, S., "Tracing the first stars with cosmic infrared background fluctuations," Nature 438, 45-50 (November 2005).

[28] Kashlinsky, A., "Cosmic infrared background and early galaxy evolution," Physics Reports 409, 361-438 (2005). 